Education \& Training | Professor Hajime Nishitani

\section{Going global:}

How a Japanese University is revolutionising English-language learning

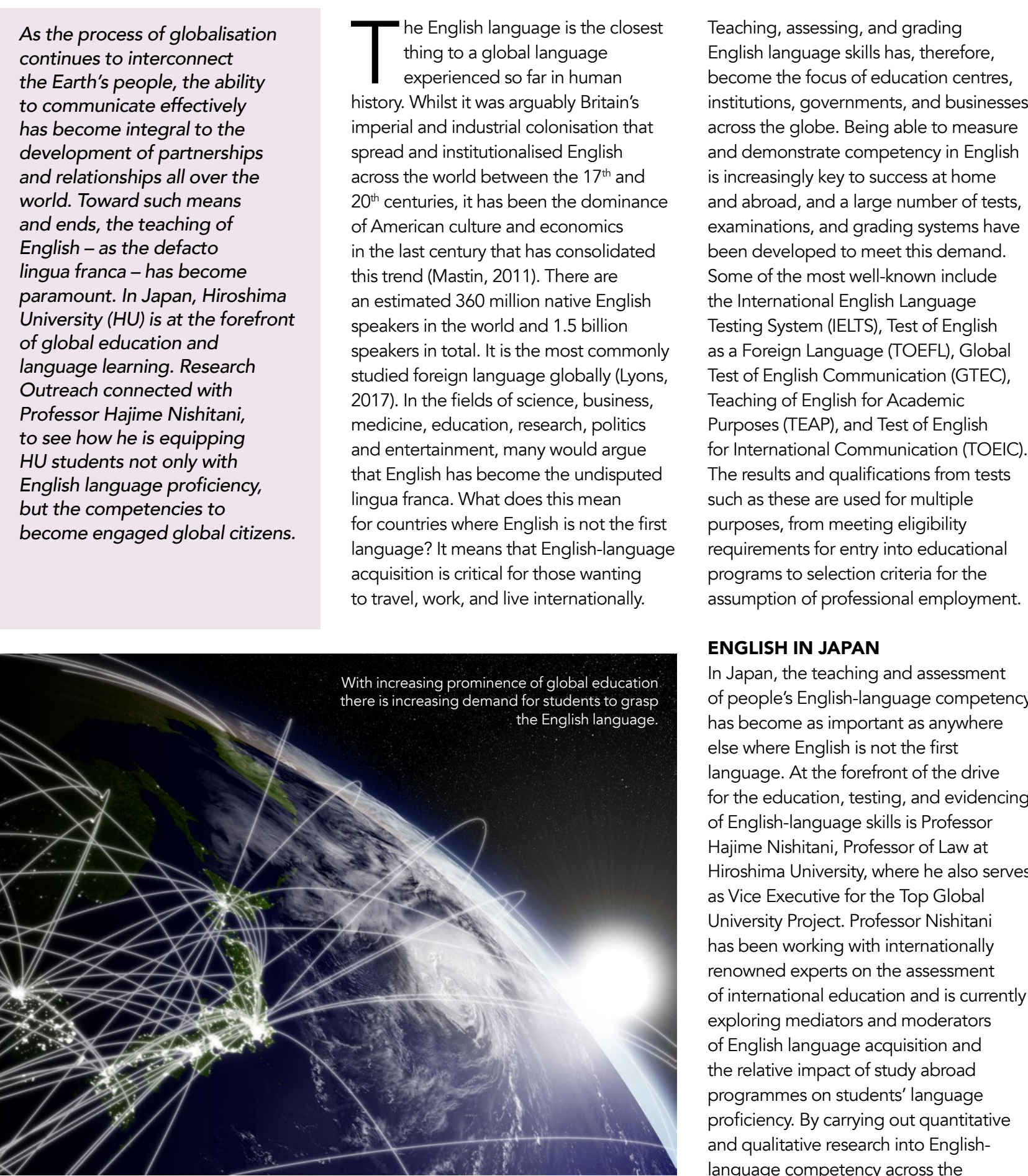

anguage competency across the

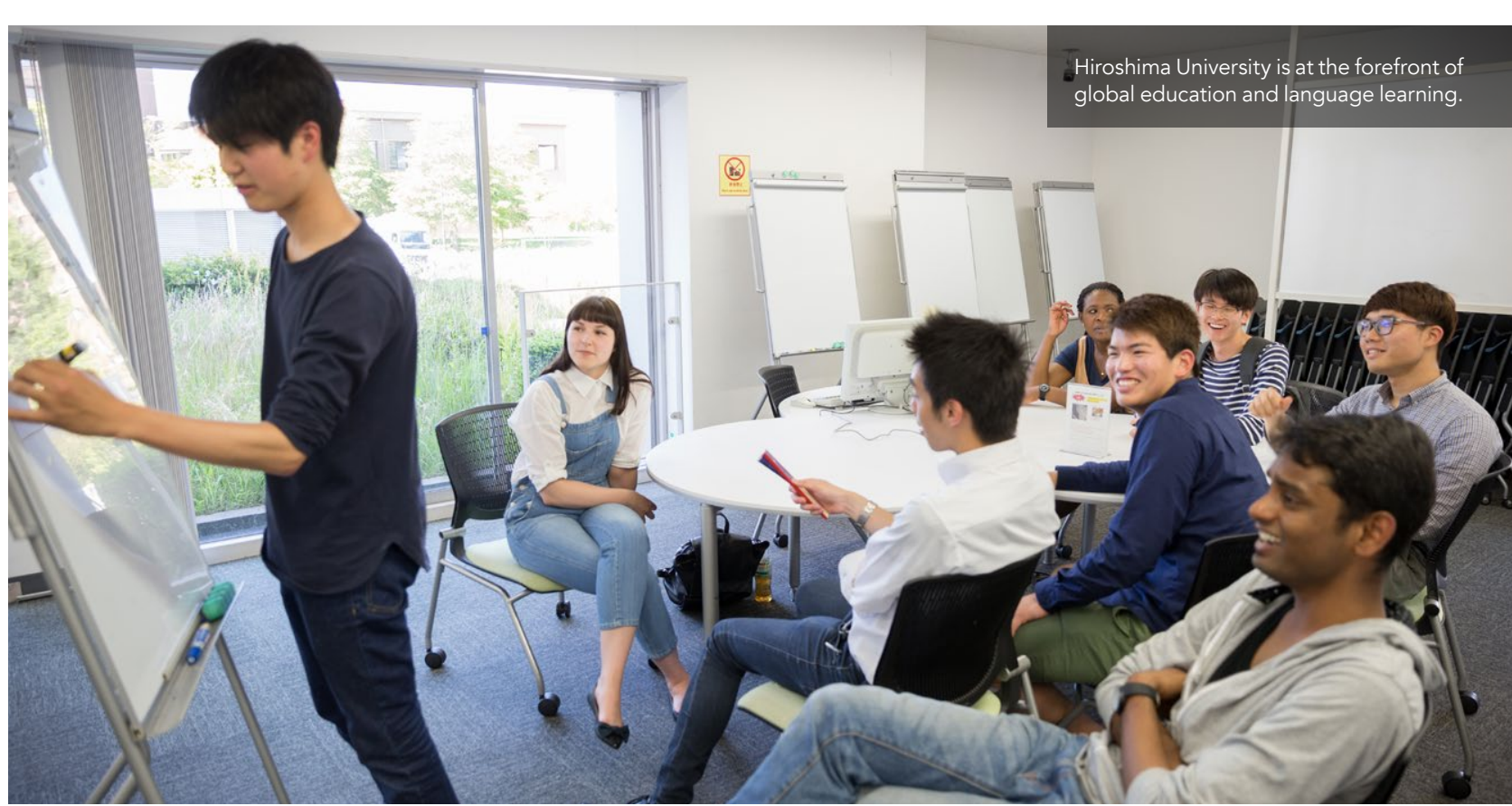

university faculties, Professor Nishitani hopes to be able to identify where the global education structure is failing students, which learning programmes are most successful, and why some students score better than others overal in testing, all with the aim of improving students' chances of success.

Since 2008, Hiroshima University has used the TOEIC Institutional Program (IP) Test to assess the English proficiency (both listening and reading) of all students. Entrance examination scores are used by the university to create target score graphs in order to allow a comparison and throughout thir sut enrolment d the that students' language skills are not improving as hoped, with some students actually receiving lower scores a year after their studies have begun. A wide variation of scores between faculties and departments has also been recorded with some score patterns repeating over more than one year, indicating potential worrying downward trends. Professor Nishitani's research specifically aims to understand why and how these scores are occurring, whether the CEFR is too broad a framework and what might be done to University students.

\section{A NEW APPROACH}

order to clarify which kind of students were improving while others were not and why, Professor Nishitani uses a grounded theory and mixed methods assessment measure called the Beliefs, Events, and The BEVI is a pory or BEVI (Shealy, 2016). can show the capacity of an individual. student's interest toward "sociocultural openness" and "global resonance" among other aspects of how individuals experience self, others, and the larger Nishitani was able to survey nearly 2,400 individual students, drawing lines and factoring inding a sudent's psychologic ent's psychologica

characteristics, department, facults, gender, type of educational programm the survey data showed that a "one size fits all" method of educating and testing students with regards to their Englishlanguage skills is not universally effective and may in fact be counterproductive.

For Professor Nishitani's study, "opennes, to experiences" is defined by scales and scores such as being high in global resonance and sociocultural openness, and low in gender traditionalism and basic

English-language acquisition is critical for those wanting to travel, work, and live internationally.

Number of students with TOEIC 730 and above

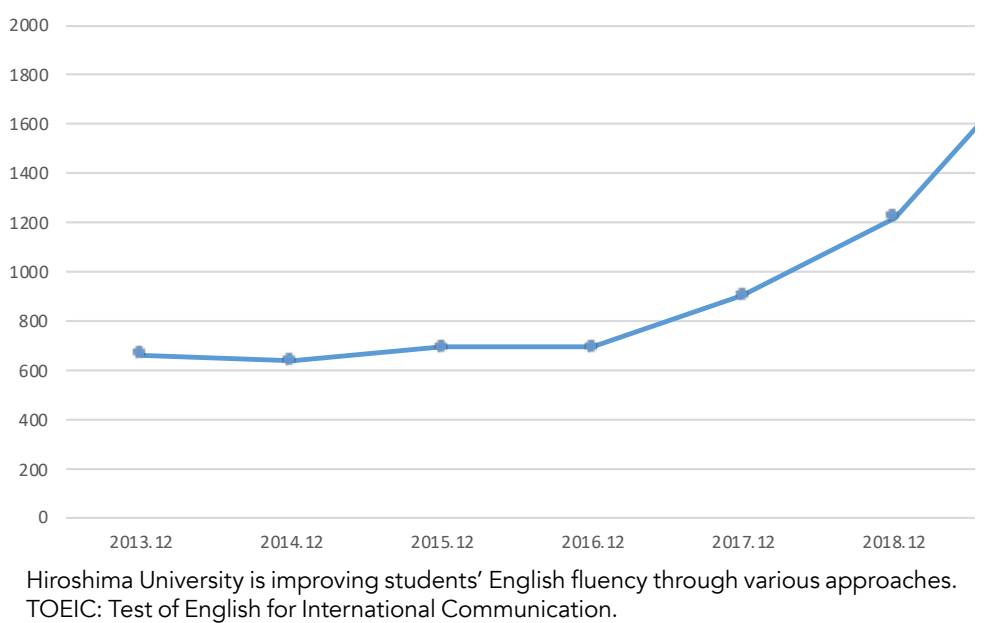


the meaning and type of BEVI scales, see www.hebev.com). Results from the survey characteristics and motivations as

measured by the $\mathrm{BEV} /$ were both distinct as well as related to their English language proficiency score. For example, students studying humanities and social studies showed a higher level of openness to new experiences compared with those studying science, technology, engineering or medical majors. There were other differences as well. For example, at the level of gender, female medical students were more open to new experiences than male medical students, with the opposite trend apparent in pharmaceutical studies. Perhaps not surprisingly, the results also showed that, almost universally, cultural openness increased following in both English and noxpish speaking countries, compared with levels measured before the visit Finally and perhaps most importantly, the results confirmed that the students who scored highly in English proficiency testing showed a higher interest in the outside world and globa engagement in general.

So how can the results of this survey prove helpful in reversing a trend of static or worsening English proficiency scores Hiroshima University and beyond? On the basis of such results, Professor Nishitan believes that the key is in treating all students as individuals and tailoring their English-language education according to their diferences. As such, he each student their own specific Englishlanguage standard/ spectationglishupon their individual motivations and

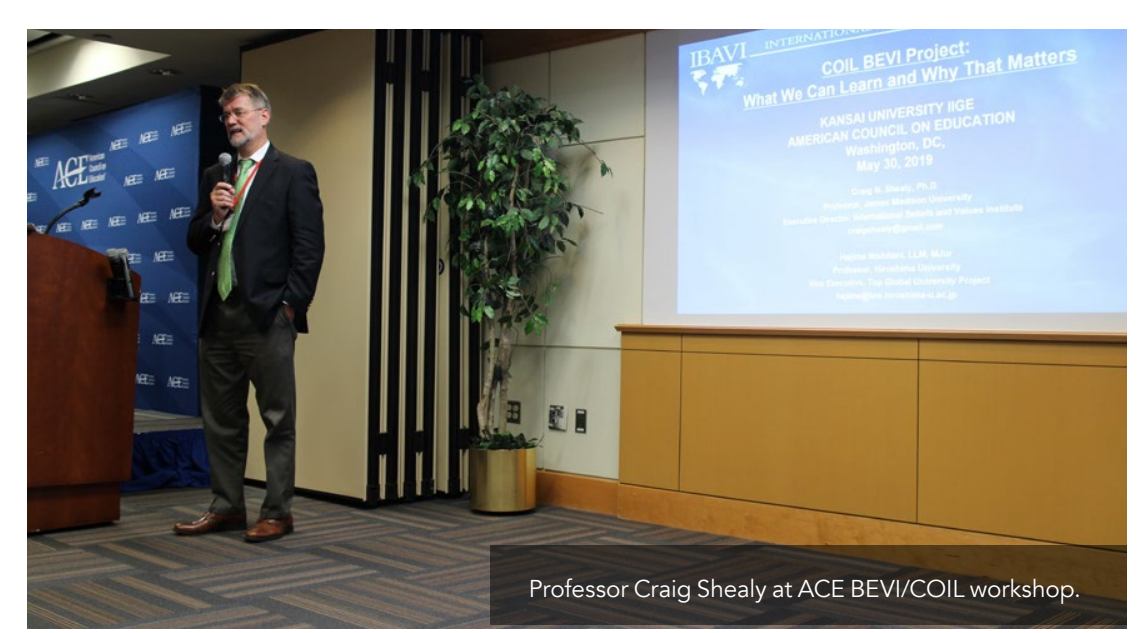

Distribution of BEVI scores by faculties and departments at Hiroshima University.

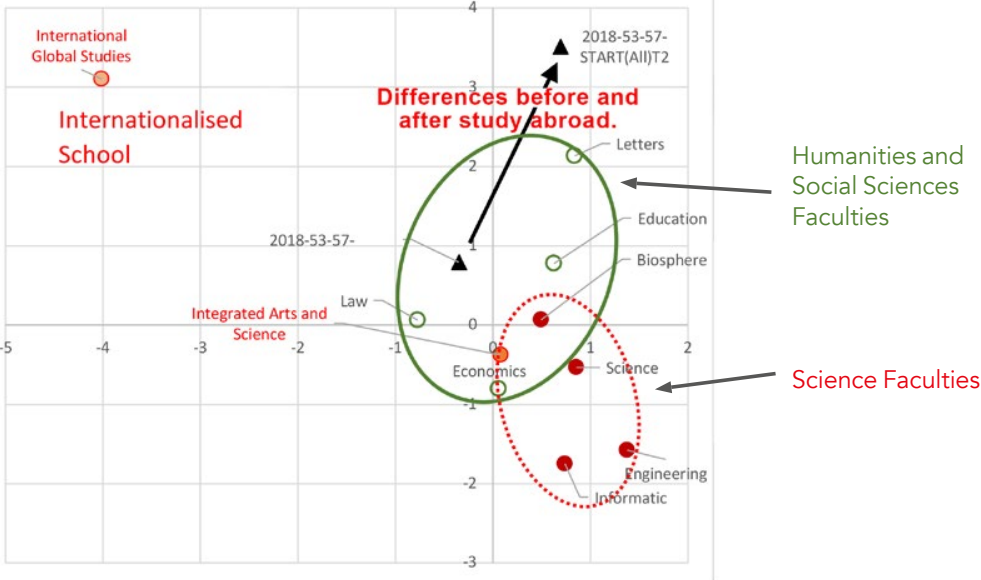

The BEVI clinical psychology-based test was carried out on Freshman Year (approx. 2,400 students)
and was conducted against faculties and departments.

Nishitani believes that the key is in treating all students as individuals and tailoring their English-language education according to their differences.

\section{interests. By employing this "expectation GLOBAL EDUCATION}

approach" as well as regularly looking

for elements to change and enhance

student motivation towards English-

language education and global activities,

the English-language learning of all students. This kind of evidence-based policy making following institutional research should lead overall to wider eduction, and berter Englin-language skills test scores, setting students up to enter the world of business or research in

In a world in which international travel, business, and education seem set to continue to increase in significance and in which the English language is the most common medium of communication, it is vital that nonEnglish speaking countries find the best ways to educate and test young peopl English. Educators and institutions represent a particular opportunity and hold a particular responsibilly to act as language acquisition in countries like Japan, and in the case of Hiroshima University, they have certainly stepped up to the plate. In fact, Professor Nishitani's research, results, and the following educational measures have already caused a stir, and together with the creator of the BEVI, Professor Craig Shealy, he has been invited to give a workshop on the topic by the American Council on Education and in other venues. Whilst the world grows smaller and globalisation continues to shape our lives, it seems safe to assume that it is those who are most globally competent and open to who survive and prosper.

E: hajime@law.hiroshima-u.ac.jp T: +81-82-424-2027

Research Objectives

Professor Nishitani's research focuses on the mediators and moderators of global engagement in general and English language proficiency in particular.

\section{Detail}

Kagamiyama 1-2-1, Higashi-Hiroshima Kiroshima, Japan $739-8525$

Hajime was previously Fulbright Professor to Harvard Law School and UC Bekeley Boalt Hall Law School. He is now Professor of Law at Hiroshima University, where he serves as Vice Executive for the Top Global University Project. Hajime collaborates with internationally renowned experts on the assessment of international learning. He currently explores the mediators and moderators of English language acquisition and the impact of study abroad.

Funding

-The Ministry of Education, Culture, Sports, Science and Technology (MEXT), JAPAN

- Japan Society for Promotion of Science (JSPS)

- Grant-in-Aid for Scientific Research (B)

Grant-in-Aid for challenging Exploratory Research Top Global University Project

\section{Collaborator}

Shealy, James Madison University, and Executive Director, International Beliefs and Values Institute (IBAVI), USA

- Professor Lee Sternberger, Associate Provost, Executive Director Center for Global Engagement, James Madison University, USA

- Professor Keisuke Kawata, Tokyo University, Japan - Professor Shinji Kaneko, Hiroshima University, Japan - Professor Mitsuyasu Morita, Hiroshima University, Japan - Professor Keiko keda, Kansai University, Japan

- Dr Emi Iwatani, Digital Promise, USA

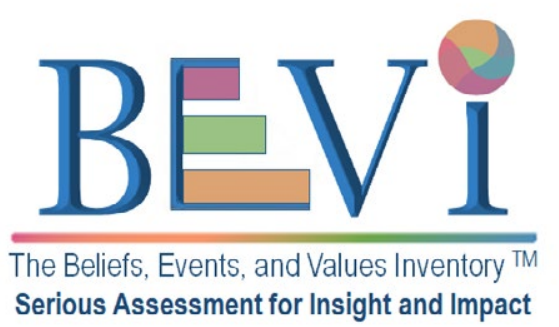

\section{References}

Lyons, D. (2017) How Many People Speak English, And Where Is It Spoken? [online] Babbel Magazine. Available at: https:// www.babbel.com/en/magazine/how-many-people-speak-

Mastin, L. (2011) Is English a Global Language? [online The History of English. Available at: https:// /www. thehistoryofenglish.com/issues global.html [Accessed 14 May

Shealy, C. N. (Ed.). (2016). Making sense of beliefs and values: Theory, research, and practice. New York: Springer Publishing A May 2019]

\section{Personal Response}

What will your workshop on this topic focus on and what do you hope to teach others about global education and English-language learning?

II BEVI is adopted as a tool to measure collaborative online international learning (COIL). And I/we are going to This weekend: Assessing Student Learning through COIL Virtual Exchanges: Overview of the BEVI Next we chend: Understanding Students' Transformational Learning

COIL is explained at the following url: https://www.acenet.edu/news-room/Pages/US-Japan_ COIL Initiative.aspx

http.//www.kansai-u.ac.jp/Kokusai/IIGE/ 